\title{
Neue Richtlinien zur Gestaltung von Manuskripten
}

\author{
Autor*innen

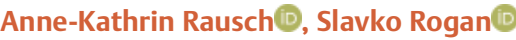 \\ Bibliografie \\ physioscience 2021; 17: 99-100 \\ DOI 10.1055/a-1544-9649 \\ ISSN 1860-3092 \\ (c) 2021. Thieme. All rights reserved. \\ Georg Thieme Verlag KG, Rüdigerstraße 14, \\ 70469 Stuttgart, Germany
}

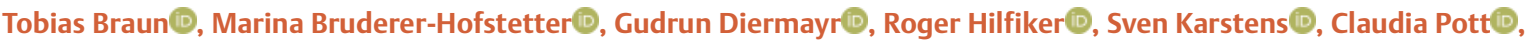

Korrespondenzadresse

Prof. Dr. Tobias Braun

Hochschule für Gesundheit Bochum

Studienbereich Physiotherapie, Gesundheitscampus 6-8,

44801 Bochum, Deutschland

tobias.braun@hs-gesundheit.de physioscience ist eine wissenschaftliche Zeitschrift mit dem Ziel, Forschungsberichte aus allen Bereichen der Physiotherapie zu publizieren, die Relevanz für Praxis, Forschung und Bildung haben. Die Zeitschrift hat sich in den letzten Jahren erkennbar weiterentwickelt und an Bedeutung gewonnen. Sie ist mit fast 8000 Exemplaren die auflagenstärkste wissenschaftliche Fachzeitschrift für Physiotherapie im deutschsprachigen Raum. Einige Herausgeber*innen sind Mitglieder der International Society of Physiotherapy Journal Editors (ISPJE). Die Kooperation mit dem Schweizer Physiotherapie Verband physioswiss, der die physioscience an alle Mitglieder versendet, besteht seit 2006. Eine bedeutsame Entwicklung in jüngster Vergangenheit ist die Etablierung der physioscience als offizielles Publikationsorgan der Deutschen Gesellschaft für Physiotherapiewissenschaft (DGPTW).

Nicht zuletzt infolge der zunehmenden Akademisierung der Physiotherapie in Deutschland, der Schweiz und Österreich hat sich die physiotherapeutische Forschung in diesen Ländern stetig weiterentwickelt. Auch international sind die absolute Zahl sowie der relative Anteil physiotherapeutischer Publikationen in der biomedizinischen Forschung in den letzten 15 Jahren konstant gestiegen [1]. Wir möchten diese äußerst positive Entwicklung unterstützen und den Autor*innen physiotherapeutischer Forschung auch zukünftig ein breites Forum für einen wissenschaftlichen Austausch bieten. Gleichzeitig möchten wir den Leser*innen aus Praxis, Forschung und Bildung eine Zeitschrift anbieten, die höchste Ansprüche an Wissenschaftlichkeit, Transparenz, Qualität, Integrität und Innovation erfüllt. Dafür ist eine konstante Weiterentwicklung der physioscience nötig, die sich an den nationalen und internationalen Entwicklungen der physiotherapeutischen und biomedizinischen Forschung orientiert.

Im Sinne dieser Weiterentwicklung haben wir den Scope der physioscience geschärft und neue Standards für die Manuskriptgestaltung eingeführt, die in den aktualisierten Richtlinien für Autor*innen formuliert sind. Diese Richtlinien sollen eine Hilfe bei der Erstellung von Manuskripten für die physioscience sein. Zudem sind wir überzeugt, dass die neuen Publikationsstandards zu einer besseren Qualität und Transparenz der Berichterstattung führen werden. Im Folgenden werden die wichtigsten Neuerungen kurz erläutert.

Die Artikelkategorien wurden überarbeitet, sodass Autor*innen nun eingeladen sind, Originalarbeiten, systematische Übersichtsarbeiten, theoretische oder methodische Grundlagenarbeiten/Analysen sowie Fallberichte einzureichen. Unter Originalarbeiten fallen z. B. klinische Studien, Beobachtungsstudien, Fallstudien, qualitative Studien oder Umfragen. Zudem sind Diskussionsbeiträge willkommen, die berufspolitische Themen aufgreifen und zum wissenschaftlichen Diskurs innerhalb der Profession und über deren Grenzen hinaus beitragen. Zu dieser Kategorie zählen Studienprotokolle und narrative Übersichtsarbeiten, deren Einreichung weiterhin möglich ist. Die neue Kategorie „Kurzberichte“ bietet Autor*innen die Möglichkeit, Ergebnisse einer Originalarbeit als Kurzbericht in der Struktur eines ausführlichen Abstracts mit maximal 2000 Wörtern einzureichen. Genaue Hinweise zu den einzelnen Artikelkategorien und den jeweiligen formalen Vorgaben finden sich in den Richtlinien für Autor*innen auf unserer Webseite. Auch weiterhin werden deutsch- und englischsprachige Arbeiten angenommen.

Leitlinien für Forschungsberichte, sogenannte Reporting Guidelines, sind einfache, strukturierte Werkzeuge, die Forschende beim Verfassen von Manuskripten verwenden sollten [2]. Eine Reporting Guideline enthält eine Liste mit Informationen, die mindestens erforderlich sind, um sicherzustellen, dass ein Manuskript von Leser*innen verstanden, von Forschenden repliziert, von Gesundheitsfachpersonen für klinische Entscheidungen genutzt und in systematische Übersichtsarbeiten eingeschlossen werden kann. Wir empfehlen allen Autor*innen, bei der Gestaltung ihrer Manuskripte mindestens eine geeignete und international anerkannte Reporting Guideline zu berücksichtigen. Das EQUATORNetzwerk (www.equator-network.org) bietet dafür eine gute Auswahl an Leitlinien.

Zukünftig werden alle Artikel den Abschnitt „Erklärungen“ enthalten, in dem die Autor*innen zu folgenden Aspekten obligatori- 
sche Angaben machen: ethische Aspekte, Zustimmung zur Veröffentlichung, Verfügbarkeit von Daten und Materialien, Studienregistrierung, finanzielle Unterstützung, Interessenkonflikte, Autor*innenbeiträge und Danksagungen. Diese Standards werden zu einer höheren Qualität der Artikel, mehr Transparenz, einer besseren Reproduzierbarkeit der Forschung und einer höheren Forschungsintegrität beitragen [3]. Wir möchten physiotherapeutische Forschung im Sinne der guten wissenschaftlichen Praxis [5] fördern und daher alle Autor*innen dazu aufrufen, ethische Prinzipien einzuhalten [6], klinische Studien und systematische Übersichtsarbeiten prospektiv zu registrieren [7] sowie Daten und Materialien bereitzustellen, die erforderlich sind, um die im Artikel berichteten Ergebnisse interpretieren, replizieren und darauf aufbauen zu können [8]. physioscience unterstützt die aktuellen Forderungen nach vertrauenswürdiger Forschung im Sinne der „Hongkong-Prinzipien“ zur Förderung von Forschungsintegrität [3]. Vertrauenswürdige Forschung ist in allen Phasen (Design, Ausführung und Berichterstattung) robust, gründlich und transparent. In diesem Zusammenhang können wir Autor*innen besonders auf der Ebene der Berichterstattung unterstützen, auf der Transparenz, die Verwendung von Reporting Guidelines und Open Access wichtige Indikatoren für verantwortungsvolle Forschungspraktiken darstellen [3].

Um eine vollständige und transparente Berichterstattung zu gewährleisten, ist die maximal zulässige Wörterzahl mitunter nicht ausreichend. Autor*innen können daher Datensätze, Tabellen, Abbildungen und andere Informationen als sogenanntes Zusatzmaterial einreichen, das zusammen mit dem akzeptierten Artikel ausschließlich online veröffentlicht wird. Auch der Peer-Review-Prozess der physioscience, ein wichtiges Qualitätskriterium wissenschaftlicher Publikationen, wird transparenter und offener. Der bisher zweiseitig verblindete Prozess wird zukünftig nur noch einseitig verblindet erfolgen. Das heißt, dass den Gutachter*innen die Identität der Autor*innen sowie die affiliierten Institutionen bekannt sind. Open-Access-Publikationen sind ebenfalls möglich. Autor*innen können auf Wunsch Beiträge in der physioscience seit einiger Zeit auch als Gold Open Access publizieren [9], wofür ein Betrag in Form einer Article Publication Charge (APC) zu entrichten ist. Alle anderen Beiträge werden im sogenannten Green Open Access publiziert. Das erlaubt es Autor*innen der physioscience, mit ihren Artikeln weltweit eine bessere Sichtbarkeit, Nutzung, Verbreitung und Zitierungen zu erlangen.

Die neuen Richtlinien für Autor*innen gelten ab 01.09.2021. Alle eingereichten Manuskripte müssen ab diesem Datum entsprechend verfasst sein. Autor*innen von Manuskripten, die sich noch in der Begutachtung oder Überarbeitung befinden, sollen die neuen Richtlinien soweit möglich berücksichtigen (z. B. Ergänzung der Deklarationen). Die Leser*innen sollten sich jedoch darauf einstellen, dass in der Übergangszeit einige Artikel veröffentlicht werden, die noch nicht den neuen Anforderungen ent- sprechen, da wir laufende Peer-Review-Verfahren nicht beeinflussen möchten.

Seit 2017 ist physioscience in der „Web of Science Core Collection" registriert. Um die internationale Sichtbarkeit und das Renommee weiter zu stärken, streben wir eine Registrierung bei MEDLINE mit Listung in der „PubMed Journal List“ an. Die Implementierung der neuen Richtlinien für Autor*innen und die damit verbundenen wissenschaftlichen Standards unterstützen diese Bestrebungen.

Abschließend möchten wir uns bei allen Autor*innen und Gutachter*innen bedanken, die in den letzten Jahren aktiv an physioscience mitgewirkt haben. Nur durch ihre Beiträge und die kritische Diskussion der eingereichten Manuskripte kann die Zeitschrift ein attraktives wissenschaftliches Forum für die Physiotherapie im deutschsprachigen Raum bieten. Die beschriebenen Veränderungen sind aus unserer Sicht ein wichtiger Schritt zur Weiterentwicklung der Zeitschrift, die den wissenschaftlichen Diskurs in der Physiotherapie in den kommenden Jahren weiterhin maßgeblich beeinflussen soll.

Interessenkonflikt

Alle Autor*innen sind Herausgeber*innen der physioscience. Die Autor*innen geben an, dass kein weiterer Interessenkonflikt besteht.

\section{Literatur}

[1] Jesus TS, Gianola S, Castellini G et al. Evolving Trends in Physiotherapy Research Publications between 1995 and 2015. Physiother Can 2020; 72 : 122-131. doi:10.3138/ptc-2018-0065

[2] Altman DG, Simera I, Hoey J et al. EQUATOR: reporting guidelines for health research. Lancet 2008; 371: 1149-1150. doi:10.1016/S01406736(08)60505-X

[3] Moher D, Bouter L, Kleinert S et al. The Hong Kong Principles for assessing researchers: Fostering research integrity. PLoS Biol 2020; 18: e3000737 doi:10.1371/journal.pbio.3000737

[4] Bouter LM, Riet GT. Empirical research must be replicated before its findings can be trusted. J Clin Epidemiol 2020. doi:10.1016/j.jclinepi. 2020.09.032

[5] Riescher G, Haas T. Verbindlich und kompakt. Der neue DFG-Kodex „Leitlinien zur Sicherung guter wissenschaftlicher Praxis“. Ordnung der Wissenschaft; 2020: 33-42

[6] Rausch A. Ethik in der Physiotherapie. Physioscience 2016; 12: 133-134. doi:10.1055/s-0035-1567136

[7] Chalmers I, Glasziou P, Godlee F. All trials must be registered and the results published. BMJ 2013; 346: f105 doi:10.1136/bmj.f105

[8] Walport M, Brest P. Sharing research data to improve public health. Lancet 2011; 377: 537-539. doi:10.1016/S0140-6736(10)62234-9

[9] Glasziou P. The role of open access in reducing waste in medical research. PLoS Med 2014; 11: e1001651 doi:10.1371/journal.pmed.1001651 